\title{
Minocycline Hydrochloride as a Potential Adjuvant to Improve Osteoconductive and Osteoinductive Properties of Bone Substitutes in an Extra-Skeletal Bone Augmentation Model: Preliminary Observations in Rats
}

\author{
Felix B. Kleine Borgmann ${ }^{3,4}$ \\ ${ }^{1}$ Department of Periodontology and Oral Surgery, Faculty of \\ Medicine, Liège, Belgium \\ ${ }^{2}$ National Center of Pathology, Laboratoire national de santé, \\ Dudelange, Luxembourg \\ ${ }^{3}$ Luxembourg Center of Neuropathology, Luxembourg \\ ${ }^{4}$ Department of Oncology, Luxembourg Institute of Health, \\ Luxembourg \\ ${ }^{5}$ Luxembourg Centre for Systems Biomedicine, University of \\ Luxembourg, Luxembourg \\ 6 Department of Human Histology and Dermatopathology, \\ University Hospital, University of Liège, Liège, Belgium
}

Bob Biewer ${ }^{1,2, \odot}$ Dorien Van Hede ${ }^{1}$ Eric Rompen ${ }^{1} \quad$ Michel Mittelbronn $^{2,3,4,5} \quad$ Pascale Quatresooz $^{6}$

Address for correspondence Bob Biewer, MD, Route d'Arlon 95, 8211 Mamer, Luxembourg (e-mail: bob.biewer@dentist.lu).

Eur Dent Res Biomater J 2021;2:1-5.

\begin{abstract}
Keywords

- bioactive glass

- deproteinized bovine bone mineral

- minocycline hydrochloride

- guided bone augmentation

- extraskeletal bone formation

- bone histology

Objectives The present study was performed to determine if minocycline $\mathrm{HCl}$ could influence the behavior of deproteinized bovine bone mineral (DBBM) and bioactive glass (BG) particles when used as filler material for new bone generation in a guided bone augmentation model.

Materials and Methods Two occlusive titanium caps were placed on the rat calvaria. One filled with BG particles, the second with DBBM particles, both previously mixed with blood (control). In minocycline $\mathrm{HCl}$ loaded groups (experimental), grafts were additionally placed into a minocycline solution. Samples were harvested after 4,8 , and 16 weeks. Half of the samples were embedded in methylmethacrylate for undecalcified histology and the other half was fixed, decalcified, and embedded in paraffin for classical histologic analysis.

Results The control groups highlighted osteoconductive and osteoinductive responses associated to BG particles, as well as an osteoconductive reaction, in DBBM sections. The addition of minocycline $\mathrm{HCl}$ to $\mathrm{BG}$ particles had no measurable influence on the result. In minocycline $\mathrm{HCl}$ loaded DBBM sections; however, areas of spontaneous ossification could be observed after 8 and 16 weeks.

Conclusion Our observations suggest that minocycline $\mathrm{HCl}$ may add some osteoinductive properties to DBBM within the limitations of this study design. Further investigations are needed to refine the present results.
\end{abstract}

DOI https://doi.org/ 10.1055/s-0041-1727292 (c) 2021. European Dental Research and Biomaterials Journal. This is an open access article published by Thieme under the terms of the Creative Commons Attribution-NonDerivative-NonCommercial-License, permitting copying and reproduction so long as the original work is given appropriate credit. Contents may not be used for commercial purposes, or adapted, remixed, transformed or built upon. (https://creativecommons.org/licenses/by-nc-nd/4.0/). Thieme Medical and Scientific Publishers Pvt. Ltd. A-12, 2nd Floor, Sector 2, Noida-201301 UP, India 


\section{Introduction}

Peri-implant diseases are defined as infectious-inflammatory processes affecting all tissues around an osseointegrated implant, resulting in a loss of the supporting alveolar bone. Conventional treatment approaches, successful in periodontal diseases, have been proven to be insufficient to manage peri-implantitis. ${ }^{1}$ The adjunctive use of chemical agents as local or systemic antibiotics and regenerative treatment procedures have been advocated to repair such peri-implant defects. ${ }^{2,3}$

Minocycline $\mathrm{HCl}$ is a semisynthetic tetracycline of the second generation with documented additional nonantibiotic properties as an inhibition of bone resorption ${ }^{4}$ and a stimulation of bone formation. ${ }^{5,6}$ Even if unfavorable effects on bones have been described, ${ }^{7}$ tetracyclines are still explored to improve the outcome and the predictability of GBR procedures.

The approach of the guided bone augmentation (GBA) technique is to create a secluded volume around the bone defect, filled with bone grafts, or bone graft substitutes for bone regeneration without competition from other tissues. Biologically active glasses (BG) are an important class of grafting materials in bone regeneration. ${ }^{8,9}$ Biogran $^{\mathrm{TM}}$ is an easy to handle BG of the first generation, with a particle size within the 300 to $355 \mu \mathrm{m}$ range, considered as ideal for such clinical application. ${ }^{10}$

Deproteinized bovine bone mineral (DBBM) is a xenogeneic graft material that has been extensively tested and documented in scientific literature. ${ }^{11}$ The nonsintered form, free of organic matrix is by far the most clinically used bovine mineral. DBBM, so as Bio-Oss ${ }^{\circledR}$ used in this study, showed osteoconductive properties by promoting cellular adhesion, wound healing, and the formation of new bone tissue. ${ }^{12}$

The aim of the present study was to investigate whether the addition of minocycline $\mathrm{HCl}$ may have an adjuvant effect on the behavior of BG and DBBM for enhanced results in guided bone regeneration procedures.

\section{Materials and Methods}

The surface microtopography of the different bone substitute particles was examined in high vacuum using a scanning electron microscope and was imaged in a native, uncoated state at $10 \mathrm{kV}$.

The study was performed on a total of 20 adult male Wistar rats. All experimental procedures used in this investigation were reviewed and approved by the Institutional Animal Care and Use Ethics Committee of the University of Liège, Belgium, following the national and European legislation on this issue. The rats were acquired from the University of Liège. The study protocol included animal accommodation with water ad libitum, regular rat pellet with artificial light with a night and day cycle.

The animals were anaesthetized with a combination of $0.25 \mathrm{mg} / \mathrm{kg}$ of atropine sulfate, $0.5 \mathrm{~mL} / \mathrm{kg}$ of xylazine at $2 \%$ and $25 \mathrm{mg} / \mathrm{mL}$ of ketamine administered intraperitoneally. A midline incision was made on the forehead of the rat and two hollow occlusive titanium caps were subperiosteally implanted on the calvaria. One was filled with BG particles (Biogran-Biomet 3i, Palm Beach, Florida, United States) and the second with DBBM particles (Bio-Oss-Geistlich, Wolhusen, Switzerland). Bone filler particles were mixed with blood, collected from the animal's tail, and a drop of balanced salt solution to acquire a moldable consistency of the particles and a perfectly adapted fill of the regeneration chambers. The animals were randomly assigned to one minocycline treated group and one non-minocycline treated control group. In control group, only the surgical procedure was applied with no additional treatment. In minocycline $\mathrm{HCl}$ group $(n=10)$, bone grafts were additionally placed into a minocycline solution at $0.1 \mathrm{mg} / \mathrm{mL}$ prior placement.

Rats were euthanized by an overdose of pentobarbital after sedation with ketamine and xylazine 4,8 , and 16 weeks after surgery ( $n=3$ for weeks 4 and $8 ; n=4$ for week 16). The skullcap was removed and the samples were fixed for 48 hours in $10 \%$ formaldehyde and dehydrated in a graded alcohol solution.

For each observation time, two of the fixed biopsies were embedded in methylmethacrylate resin with a Giemsa, Paragon, and a combination of Stevenel's blue with Van Gieson staining for undecalcified sections. The remaining samples were decalcified in ethylenediaminetetraacetic acid (EDTA) and the paraffin-embedded blocks were toluidine blue stained for classical histologic analysis.

\section{Results}

Scanning electron microscopy (SEM) analysis of Biogran ${ }^{\mathrm{TM}}$ granules revealed a crystalline structure with a microporous surface at higher magnification. Bio-Oss $₫$ particles harbored a more porous microtopography preserving at higher magnification mostly the typical structure natural cancellous bone ( - Fig. 1).

All animals recovered well from anesthesia and surgery. No adverse reactions or device exposures were observed; all biopsies were available for analysis.

\section{4-Week Observations}

BG granules were embedded in a loose connective tissue and active angiogenesis was observable. The outer shell of the

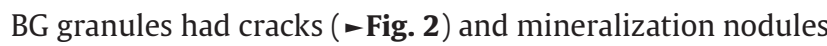
were detectable in the central part. First ectopic mineralization foci could be observed at some distance from the BG particles.

Both DBBM series presented a loose connective tissue associated to neovascularization. There were no notable differences between untreated DBBM and DBBM-minocycline loaded samples. Slight new bone formations growing along the inner wall of the titanium cap and some mineralization processes on the DBBM particles adjacent to the calvaria.

\section{8-Week Observations}

In BG series, there were some isolated areas of mineralization spots inside the cap, lined by a layer of osteoblasts ( - Fig. $\mathbf{3}$ ). 
In DBBM series, new bone was confined to the lower part of the capsule in direct contact to the calvaria. The remaining volume was mainly occupied by DBBM particles still embedded in connective tissue. In minocycline treated samples, there were some dispersed areas of spontaneous ossification not in direct contact to DBBM particles and comparable to them found in BG samples (-Fig. 4).

\section{6-Week Observations}

In BG series, larger ossification areas were observed while glass particles showed a tendency to disintegrate. The addition of minocycline $\mathrm{HCl}$ did not appear to influence neither the behavior of BG nor the new bone generation.

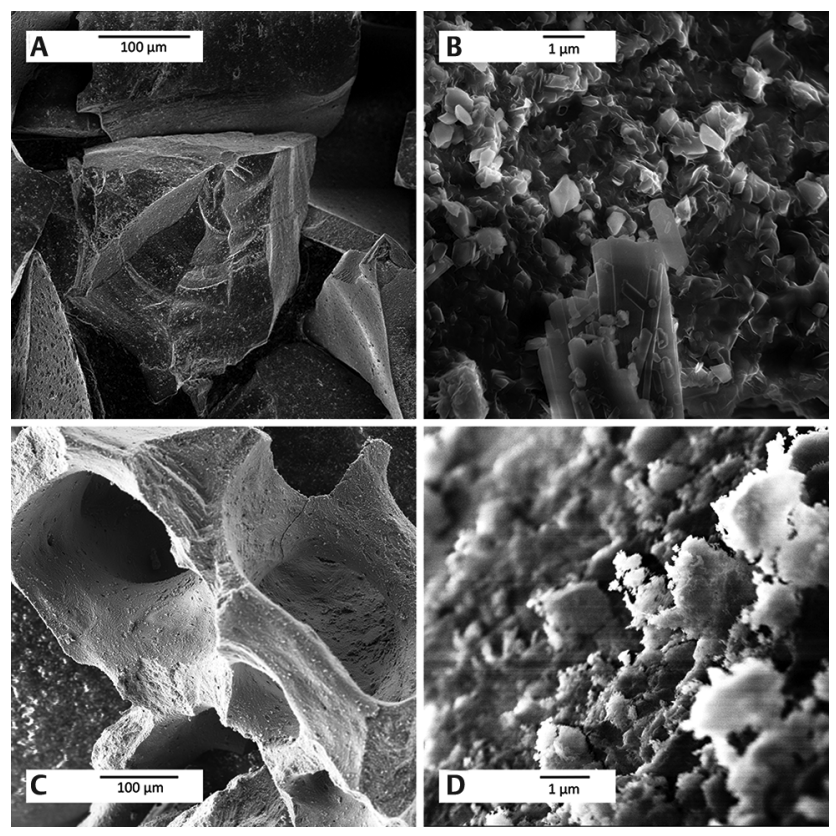

Fig. 1 (A) Scanning electron microscopy (SEM) of bioactive glass particles $\left(\right.$ Biogran $\left.^{\mathrm{TM}}\right)$. (B) Detailed view of the microporous structure of the $45 \mathrm{~S} 5$ bioactive glass particle at higher magnification. Original magnification $\times 1,000$. (C) SEM Image showing a Bio-Oss $®$ particle with a more porous microtopography preserving mostly the typical structure natural cancellous bone, original magnification $\times 1,000$. (D) Detail of the surface at higher magnification.

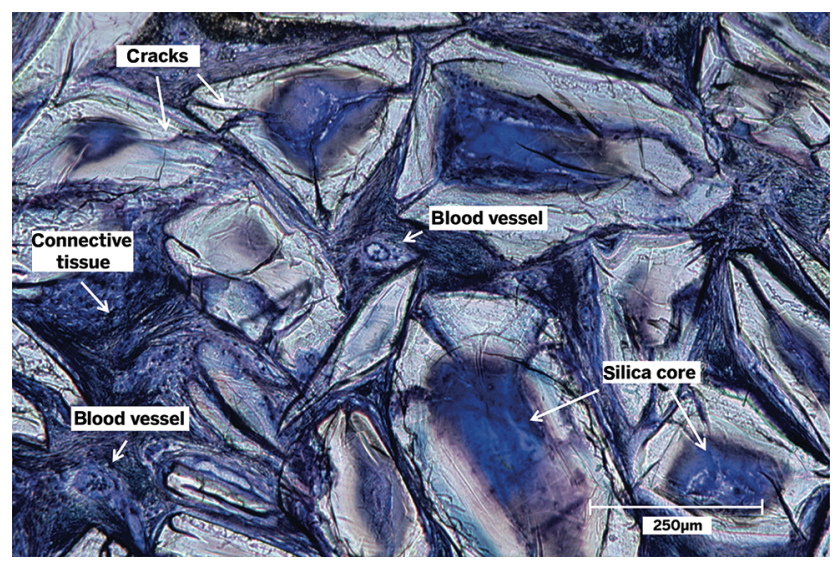

Fig. 2 Biogran $^{\mathrm{TM}}$ at 4 weeks. Cracks thorough the glass particle. The silica core tends to disappear leaving a calcium phosphate pouch. Signs mineralization at the inner part of the particle. Angiogenesis within the intergranular connective tissue. Stevenel's blue with Van Gieson staining. Magnification $\times 20$.
The majority of the DBBM graft particles was still encapsulated in fibrous tissue. In the central part of the capsule of the minocycline loaded DBBM samples, however, we found large zones of ossification embedding graft particles (-Fig. 5A and 5B). There were no visible signs of resorption of the graft particles.

\section{Discussion}

The aim of this study was to evaluate if minocycline $\mathrm{HCl}$ could influence the behavior of BG and DBBM in a GBA model.

The titanium caps used in the present study are appropriate in terms of biocompatibility and structural support to maintain the volume of the regeneration. ${ }^{13}$ The transmembranous vascularization and nutrient transfer are important factors in the process of tissue regeneration. ${ }^{14}$ The occlusive and stiff barrier provided by the caps used in this study may have been a disadvantage and can explain the relative amount of fibrous tissue we have noted. The close spatial and temporal correlation between angiogenesis and new

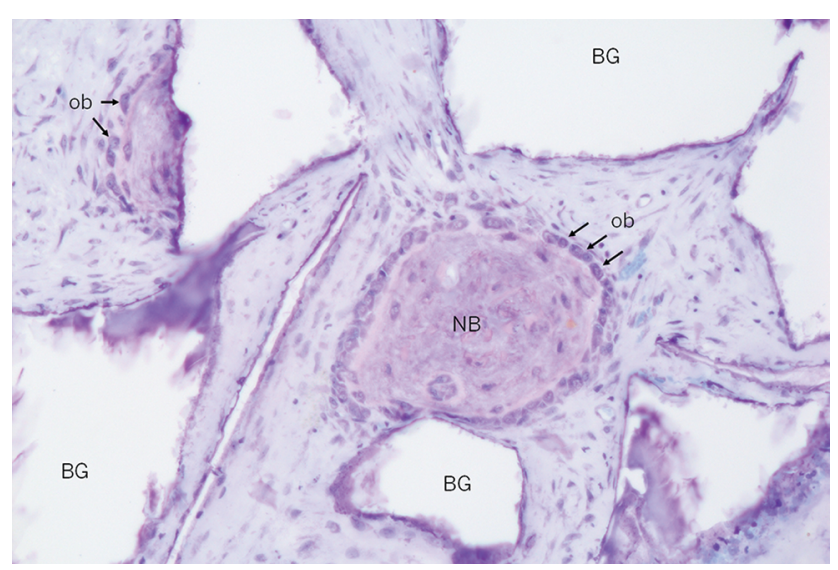

Fig. 3 Biogran $^{\mathrm{TM}}$ at 8 weeks (BG). Ossification islets (NB) lined by a layer of osteoblasts (ob) acting an osteoblastic front to centrifugal expansion (arrows). Toluidine blue staining. Magnification $\times 20$. BG, bioactive glass.

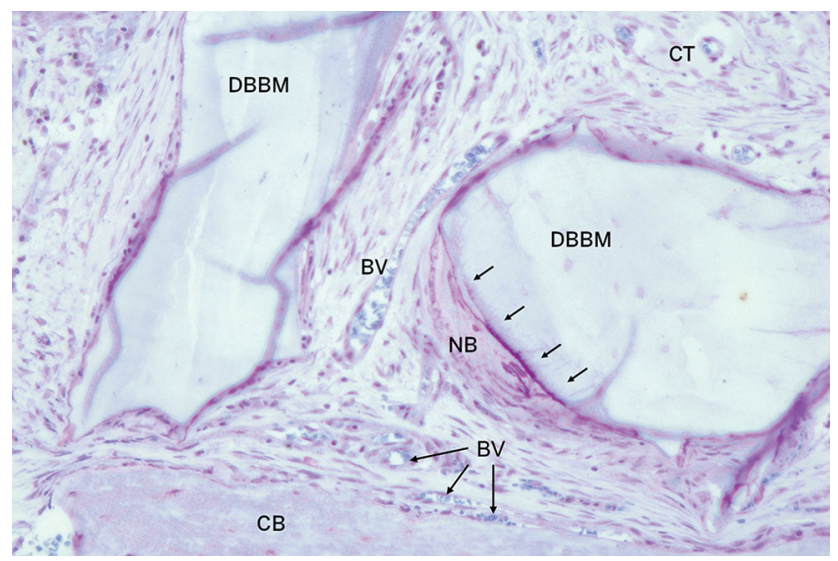

Fig. 4 Bio-Oss ${ }^{\circledR}$ at 8 weeks. Mineralization (NB) at the surface of the DBBM particles (arrows) at the bottom, close to the calvaria (CB). Graft particles embedded in loose connective tissue (CT) with new blood vessels (BV). Toluidine blue staining. Magnification $\times 20$. DBBM, deproteinized bovine bone mineral. 


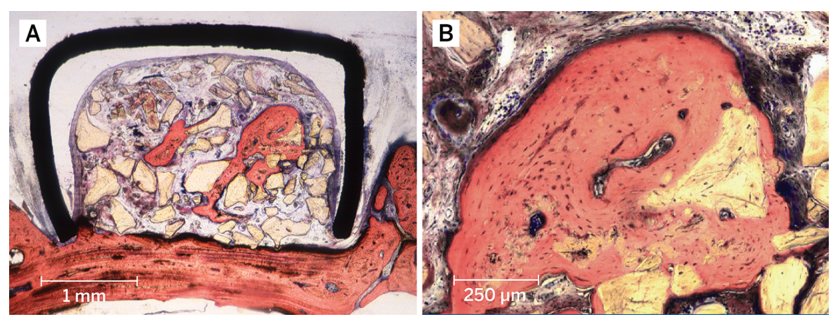

Fig. 5 (A) Bio-Oss $®$ loaded with minocycline at 16 weeks. An osteoinductive effect is observed. 20-40 $\mu \mathrm{m}$ undecalcified section. Magnification $\times 2.5$. (B) Detail of the osteoinductive and osteoconductive effect of the minocycline loaded grafting material with no signs of resorption at higher magnification; magnification $\times 20$. Giemsa, Paragon and Stevenel's blue in combination with Van Gieson's picrofuchsin stain (A and $\mathbf{B}$ ).

extraskeletal bone formations in GBA procedures have been documented. ${ }^{15}$ From a clinical point of view, this hypothesis might also be supported by the fact that some authors have recommended a perforation of adjacent cortical bone during onlay bone augmentation procedures to open bone marrow spaces which in turn might ameliorate bone formation. ${ }^{15,16}$ In this study, the calvaria was left intact, so that neither the overlying periosteum nor any opening of the marrow spaces could have had any influence on the outcome in terms of tissue formation. It should be noted that the calvaria differs in its development from the alveolar bone where clinical interventions using this technique usually are applied, which, together with the use of rat rather than human tissue, further limits the direct applicability of this study to the clinic and makes further studies necessary.

Although many studies have proved the osteoconductivity and predictability of DBBM, some published studies and case reports found that this grafting material inhibited new bone formation. ${ }^{17}$ The major portion of the capsules in this study was occupied by DBBM particles embedded in loose fibrovascular connective tissue, quite similar to the above-mentioned findings. When comparing the present observations to earlier works using the same study design and leading to $172.8 \%$ of bone augmentation in empty capsules, ${ }^{16}$ our findings seem to indicate that new bone formation has negatively been affected by the DBBM graft.

The resorption of DBBM is a controversial issue. Our observations did not reveal any evidence resorption and are in line with other research findings. ${ }^{18}$

Studies with narrow-range-sized BG particles had demonstrated the highly osteoconductive response of this material in new bone formation. ${ }^{19}$ The superficial microcracks are probably related to the corrosion of glass due to the soaking effect in contact with blood and body fluids. They allow cells to penetrate the interior silica gel, creating an ideal environment for new bone formation. ${ }^{20,21}$ The mineralization zones observed inside the dissociated particles seem to be in line with findings, showing that BG particles could act as a kind of nucleus for new bone formation. ${ }^{20}$ Our observations confirm the biocompatibility of this material and highlight its osteoconductivity and osteoinduction in form of ectopic mineralized nodules at some distance of the BG particles regardless of the treatment. The administration of minocycline $\mathrm{HCl}$ to BG did apparently not affect the number nor the distribution and maturation of these mineralization areas.

The release of the antibiotic from the graft materials depends not only on the type and the concentration of the drug but also on the surface characteristics of the used grafting material. The adsorption kinetics and the level of porosity and surface area of the graft are probably key factors. ${ }^{22}$ The minocycline concentration and immersion time, we had chosen in this study, which gave a sufficient safety margin on fibroblasts and osteoblasts, essential as minocycline-like other derivates, have been reported to be cytotoxic above certain concentrations. ${ }^{23}$ Further investigations need to be conducted to control and quantify the concentration of the drug on the graft surface. The relatively smooth surface and poor porosity of BG particles may explain the lack of observable influence of minocycline on all BG-filled samples compared with the more crystalline calcium-rich DBBM granules.

Our findings in DBBM-only samples are in line with previous results where no osteoinductive effect has been noted. ${ }^{24}$ The newly formed bone spots in minocycline-loaded samples may have bridged the gaps between the different DBBM particles to form an extended zone of ossification independent form the original bone and indicating a possible osteoinductive effect. However, as the sectioning protocol limits the possibility to look at adjacent areas, a potential association of the "osteo-induced" centers with adjacent "osteo-conducted" bone formations in relation with the basal skull bone situated perhaps outside of our observation fields cannot be excluded.

\section{Conclusion}

This study may have clinical interest in the treatment of bone infections and bone defects such as in cases of peri-implantitis. Minocycline $\mathrm{HCl}$ may be considered as a promising complementary treatment approach. The observations of the present study confirm that DBBM particles were found to be osteoconductive and, in combination with minocycline, potentially osteoinductive. The osteoconductive and osteoinductive properties of bioactive glass particles could be confirmed within the study limitations. Further investigations with a more suitable study design are needed to refine the present results.

\section{Conflict of Interest}

None declared.

\section{References}

1 Schwarz F, Papanicolau P, Rothamel D, Beck B, Herten M, Becker J. Influence of plaque biofilm removal on reestablishment of the biocompatibility of contaminated titanium surfaces. J Biomed Mater Res A 2006;77(3):437-444

2 Ericsson I, Persson LG, Berglundh T, Edlund T, Lindhe J. The effect of antimicrobial therapy on periimplantitis lesions. An experimental study in the dog. Clin Oral Implants Res 1996;7(4):320-328 
3 Zafar MS, Farooq I, Awais M, Najeeb S, Khurshid Z, Zohaib S. Bioactive surface coatings for enhancing osseointegration of dental implants. In: Biomedical, Therapeutic and Clinical Applications of Bioactive Glasses. Cambridge, United Kingdom: Woodhead Publishing; 2018

4 Gomes PS, Fernandes MH. Effect of therapeutic levels of doxycycline and minocycline in the proliferation and differentiation of human bone marrow osteoblastic cells. Arch Oral Biol 2007;52(3):251-259

5 Williams S, Wakisaka A, Zeng QQ, et al. Effect of minocycline on osteoporosis. Adv Dent Res 1998;12(2):71-75

6 Bain S, Ramamurthy NS, Impeduglia T, Scolman S, Golub LM, Rubin C. Tetracycline prevents cancellous bone loss and maintains near-normal rates of bone formation in streptozotocin diabetic rats. Bone 1997;21(2):147-153

7 Simmons DJ, Chang SL, Russell JE, Grazman B, Webster D, Oloff C. The effect of protracted tetracycline treatment on bone growth and maturation. Clin Orthop Relat Res 1983;(180):253-259

8 Khurshid Z, Husain S, Alotaibi H, et al. Novel techniques of scaffold fabrication for bioactive glasses. In: Biomedical, Therapeutic and Clinical Applications of Bioactive Glasses. Cambridge, United Kingdom: Woodhead Publishing; 2018

9 Skallevold HE, Rokaya D, Khurshid Z, Zafar MS. Bioactive glass applications in dentistry. Int J Mol Sci 2019;20(23):1-24

10 Wheeler DL, Eschbach EJ, Hoellrich RG, Montfort MJ, Chamberland DL. Assessment of resorbable bioactive material for grafting of critical-size cancellous defects. J Orthop Res 2000;18(1):140-148

11 Aludden HC, Mordenfeld A, Hallman M, Dahlin C, Jensen T. Lateral ridge augmentation with Bio-Oss alone or Bio-Oss mixed with particulate autogenous bone graft: a systematic review. Int J Oral Maxillofac Surg 2017;46(8):1030-1038

12 Hämmerle $\mathrm{CH}$, Karring T. Guided bone regeneration at oral implant sites. Periodontol2000 1998;17:151:175

13 Zafar MS, Fareed MA, Riaz S, Latif M, Habib SR, Khurshid Z. Customized therapeutic surface coatings for dental implants. Coatings 2020;10(6)

14 Schwarz F, Rothamel D, Herten M, Sager M, Becker J. Angiogenesis pattern of native and cross-linked collagen membranes: an immunohistochemical study in the rat. Clin Oral Implants Res 2006;17(4):403-409
15 Schmid J, Hämmerle CHF, Flückiger L, et al. Blood-filled spaces with and without filler materials in guided bone regeneration. A comparative experimental study in the rabbit using bioresorbable membranes. Clin Oral Implants Res 1997;8(2):75-81

16 Rompen EH, Biewer R, Vanheusden A, Zahedi S, Nusgens B. The influence of cortical perforations and of space filling with peripheral blood on the kinetics of guided bone generation. A comparative histometric study in the rat. Clin Oral Implants Res 1999;10(2):85-94

17 Stavropoulos A, Kostopoulos L, Mardas N, Nyengaard JR, Karring T. Deproteinized bovine bone used as an adjunct to guided bone augmentation: an experimental study in the rat. Clin Implant Dent Relat Res 2001;3(3):156-165

18 Valentini P, Abensur D, Densari D, Graziani JN, Hämmerle C. Histological evaluation of Bio-Oss in a 2-stage sinus floor elevation and implantation procedure. A human case report. Clin Oral Implants Res 1998;9(1):59-64

19 Ducheyne P, Hench LL, Kagan A II, Martens M, Bursens A, Mulier JC. Effect of hydroxyapatite impregnation on skeletal bonding of porous coated implants. J Biomed Mater Res 1980;14(3):225-237

20 Schepers E, de Clercq M, P Ducheyne, R Kempeneers. Bioactive glass particulate material as a filler for bone lesions. J Oral Rehabil 1991;18(5):439:452

21 Veis AA, Dabarakis NN, Parisis NA, Tsirlis AT, Karanikola TG, Printza DV. Bone regeneration around implants using spherical and granular forms of bioactive glass particles. Implant Dent 2006;15(4):386-394

22 Saito T, Takeuchi R, Hirakawa K, et al. Slow-releasing potential of vancomycin-loaded porous hydroxyapatite blocks implanted into MRSA osteomyelitis. J Biomed Mater Res 2002;63(3):245-251

23 Rompen EH, Goffinet GH, Nusgens B. Human periodontal ligament fibroblast behavior on chemically conditioned dentine: an in vitro study. J Periodontol 1999;70(10):1144-1152

24 Hockers T, Abensur D, Valentini P, Legrand R, Hammerle CHF. The combined use of bioresorbable membranes and xenografts or autografts in the treatment of bone defects around implants. A study in beagle dogs. Clin Oral Implants Res 1999;10(6):487-498 